\title{
Permanencia en educación universitaria en Perú
}

\author{
Análisis en el marco del aseguramiento de condiciones \\ básicas de calidad
}

\author{
Fiorella Castro Aguirre ${ }^{1}$
}

\begin{abstract}
This research presents an empirical analysis of the decisions of permanence by university students in the context of the implementation of the reform started in 2014 in Peru. One of the pillars of this reform is the granting of institutional licenses after the verification of up to eight basic quality conditions in the provision of the educational service offered by the universities. In this ongoing process, there is a growing concern about the possibilities that students could continue with their studies after the denial of licenses to universities, programs and / or affiliates. Using information on educational status, characteristics of individual students and of their homes, as well as characteristics of the educational offer (type of management of the university, the cost of the educational service and obtaining the institutional license), a discrete choice model for panel date is estimated. Results show evidence on the factors that determine the permanence of university students. Among the most important factors are: (i) student performance, (ii) participation in the labor market, (iii) a proxy for university quality (cost of educational service) and (iv) the type of management of the university.
\end{abstract}

Keywords: University reform, permanence, human capital, discrete choice.

\section{RESUMEN}

Esta investigación presenta un análisis empiríco de las decisiones de permanencia de los estudiantes universitarios en el contexto de la implementaci ón de la reforma universitaria iniciada en el 2014 en Perú. Uno de los pilares de esta reforma es el otorgamiento de licencias institucionales, luego de la verificación de hasta ocho condiciones básicas de calidad en la prestación del servicio educativo que ofrecen las universidades. En este proceso en curso, existe una creciente preocupación por las posibilidades de que los estudiantes puedan continuar con sus estudios universitarios frente a la denegación de las licencias a universidades, programas y/o filiales. Utilizando información sobre el estado educativo, características individuales de los estudiantes y de sus hogares, así como características de la oferta educativa (tipo de gestión de la universidad, el costo del servicio educativo y obtención de la licencia institucional), se estima un modelo de elección discreta con datos de panel, cuyos resultados muestran evidencia sobre los factores que determinan la permanencia de los estudiantes universitarios. Entre los factores más importantes se encuentran: (i) el desempeño de los estudiantes, (ii) la participación en el mercado laboral, (iii) un proxy de la calidad universitaria (costo del servicio educativo) y (iv) el tipo de gestión de la universidad.

Palabras clave: Reforma universitaria, permanencia, capital humano, elección discreta.

Received: 15 de abril del 2020

Accepted: 15 de mayo de 2020

\section{Introducción}

La educación superior permite contar con capital humano calificado, el cual contribuye la mejorar la productividad y el crecimiento económico. Bajo el análisis del capital humano, este incrementa los ingresos individuales y la productividad, a través del conocimiento y las habilidades adquiridas (Becker, 1994). A lo largo del siglo XX y lo que va del presente siglo, se ha registrado una notable expansión de la educación superior a nivel mundial, tanto en países desarrollados como en países en desarrollo.

En América Latina la tasa promedio de matrícula en la educación superior se incrementó rápidamente durante las últimas décadas; de $17 \%$ a $21 \%$ y después a $43 \%$ en los años 1991, 2000 y 2013, respectivamente (Ferreyra et al., 2017). Los países de la región optaron por el financiamiento privado para atender la mayor demanda de educación superior, debido a las recomendaciones de los organismos internacionales en los años 90 (Rodríguez y Montoro, 2013). Asimismo, la mayor expansión alcanzada en la década de los 2000 se explica por las políticas implementadas como las becas y créditos educativos. En este contexto, el incremento de las instituciones de educación superior o universidades estuvo asociado con la provisión de un servicio a menores costos (permitiendo una mayor cobertura a segmentos de menores ingresos), pero con calidad discutible.

Adicional al acceso y permanencia, los estudios resaltan la importancia de la calidad del servicio educativo. Así, el Programa de las Naciones Unidades para el Desarrollo (PNUD 2015), en los Objetivos de Desarrollo Sostenible 2015-2030, declara como Objetivo № 4: "Garantizar una educación inclusiva, equitativa y de calidad y promover oportunidades de aprendizaje durante toda la vida para todos"; siendo una de sus metas para el 2030, asegurar el acceso en condiciones de igualdad para todos los hombres y las mujeres a una formación técnica, profesional y superior

\footnotetext{
Economista por la Universidad Nacional del Callao, y Magister en Economía por la Universidad Torcuato di Tella. Especialista en evaluación de políticas sociales del Ministerio de Desarrollo e Inclusión Social, Perú. Email:fcastro@midis.gob.pe. Las opiniones expresadas en el presente documento son de exclusiva responsabilidad de la autora y no representan, necesariamente, la posición de la institución donde labora.
}

How to cite: 1 INVESTIGADOR: Castro, F. (2020). Permanencia en educación universitaria en Perú: Análisis en el marco del aseguramiento de condiciones básicas de calidad. Revista de Análisis Económico y Financiero, Vol.3,N.1, 1-6. DOI: 10.15446/ing.investig.xxxx 
de calidad, incluida la enseñanza universitaria. En este aspecto algunos países la región establecieron sistemas de aseguramiento de la calidad de la educación superior; Argentina, Chile y Colombia los implementaron en la década de los 90's.

En el Perú las políticas públicas para la mejora de la calidad de la educación superior recién se materializaron en el 2014 con el inicio de la reforma universitaria. A partir de ese año, el gobierno peruano promulgó la Ley Universitaria 30220, creó la Superintendencia Nacional de Educación Superior Universitaria (Sunedu) y aprobó la Política de Aseguramiento de la Calidad de la Educación Superior Universitaria, estableciendo así el marco regulatorio para las acciones de supervisión, fiscalización y garantía de la calidad del servicio educativo.

Así, en el 2015 se estableció el mandato de cumplimiento de las condiciones básicas de calidad en las universidades para la obtención de su licencia institucional (autorización de funcionamiento). Como resultado de este proceso de licenciamiento, a la fecha de realización de este estudio 74 instituciones obtuvieron su licencia para ofrecer el servicio educativo superior universitario, mientras que el número de universidades con licencia denegada asciende a 10. Ante el futuro cierre de programas, filiales y universidades que no garanticen las condiciones básicas de calidad (CBCs), la permanencia de los estudiantes universitarios puede verse afectada significativamente si no se establecen medidas efectivas para garantizar el adecuado traslado de los estudiantes, reduciendo la formación de capital humano, aspecto que es de vital importancia para incrementar la productividad y calidad de vida en una economía en desarrollo como la peruana.

Es importante señalar que la literatura reciente señala la vigencia del debate sobre la educación superior. De acuerdo a Sánchez y Singh (2018), se observa un incremento de la importancia de la educación superior en entornos cambiantes de las estructuras económicas de los países en desarrollo. La educación superior tiene efectos en la obtención de empleo, salarios y otras variables de interés. Al respecto, Del Pozo (2018) evidencia la existencia de efectos positivos de la expansión de la oferta de universidades (desregulación) sobre los salarios y condiciones laborales de los trabajadores. Por otro lado, el trabajo de Benavides et al. (2015) señala que el sistema de educación superior peruano presenta niveles de segregación vinculados con el costo de la pensión del servicio educativo, los cuales estarían relacionados con la calidad de dicho servicio, afectando de manera negativa las desigualdades en el acceso a la educación superior.

También existe evidencia reciente de que la riqueza del hogar, localización, género, antecedentes de los padres, así como las restricciones crediticias en la secundaria en adelante ayudan a explicar las desigualdades en los niveles de acceso a la educación superior (Sánchez y Singh (2018) presentan evidencia para India, Perú y Vietnam). También es relevante resaltar el rol de la educación superior en la reducción de las desigualdades de género. Por ejemplo, Guerrero (2019) describe cómo, a pesar de que la brecha de género en acceso a educación superior en Perú tiende a cerrarse, en los hogares de ingresos más bajos las desigualdades de género aún persisten; la educación superior de los hijos varones tiene mayor prioridad debido a que las tareas domésticas son asumidas por las hijas mujeres.

La motivación de la presente investigación se centra en: (i) el contexto del aseguramiento de las CBCs requiere contar con evidencia actual de los factores de la permanencia universitaria, (ii) la mayoría de los estudios sobre educación superior en países en desarrollo utilizan datos de corte transversal y factores de acceso/permanencia limitados. A la luz de los resultados del proceso de licenciamiento en el marco de la reforma universitaria, en el Perú no se han realizado estudios que exploren las posibles relaciones (no necesariamente de tipo causal) entre indicadores de calidad del servicio educativo y variables de interés como la permanencia de los estudiantes universitarios.

En ese sentido, se plantean las siguientes preguntas: ¿Cuáles son los factores que determinan la permanencia de los estudiantes en el marco del proceso de licenciamiento de las universidades? ¿La licencia institucional, como indicador del cumplimiento de condiciones básicas de calidad, influye positivamente en la permanencia universitaria?. En particular, el presente estudio tiene por objetivo principal examinar los potenciales factores de la permanencia universitaria, en el marco del aseguramiento de condiciones básicas de calidad en la universidad peruana.

\section{Reforma universitaria y condiciones básicas de calidad}

\section{El estado de las cosas antes de la reforma}

Durante la década del 90, de manera similar a otros países de la región, en Perú se implementaron un conjunto de reformas estructurales orientadas a una mayor participación de la inversión privada en la actividad económica. Estas reformas tuvieron como sello distintivo un agresivo programa de privatización de empresas públicas, una mayor apertura comercial, y esquemas de promoción de la inversión privada a través de contratos de estabilidad tributaria. Adicionalmente, se implementaron un conjunto de políticas de estabilización y de reformas estructurales en los ámbitos monetario, cambiario, fiscal y tributario.

El proceso de privatización de empresas prestadoras de servicios públicos estuvo acompañado de la implementación de un aparato regulador y supervisor de las condiciones en las que se ofrecen estos servicios (Pasco-Font y Saavedra, 2001). Las condiciones del sistema universitario a inicios de la década de los 90 tambiénobligaron que se implementaran medidas orientadas a la fomentar la inversión privada en la educación superior.

Estas medidas incluyeron la creación, en 1995, del Consejo Nacional de Autorización del Funcionamiento de Universidades (Conafu), organo dependiente de la Asamblea Nacional de Rectores (ANR), que tenía la función de brindar las autorizaciones de funcionamiento de las nuevas universidades privadas a nivel nacional. En adición a la creación de la Conafu, en 1996 se promulgó la Ley para Promover la Inversión en Educación, a través de la cual se decretó que toda persona natural o jurídica tenía el derecho de libre iniciativa privada para organizar instituciones educativas; es decir, se permitió que las universidades privadas funcionen como empresas con fines de lucro. 
Como resultado de estos cambios, se ampliaron tanto el nivel como la diversidad de la oferta educativa universitaria, lo que se refejó en un mayor acceso a la educación superior universitaria (entre el 2004 y 2014, el número de ingresantes a universidades se duplicó de 130 mil a casi 270 mil), principamente en universidades privadas relativamente nuevas y con precios menores al promedio.

Si bien los cambios normativos contribuyeron a incrementar la cobertura y la diversidad del sistema universitario, no se generaron los incentivos adecuados para garantizar la calidad del servicio educativo, aspecto que generó una preocupación creciente. Así, luego de 10 años de vigencia de la Ley para Promover la Inversión en Educación, se creó, en el año 2006, el Sistema Nacional de Evaluación, Acreditación y Certificación de la Calidad Educativa (Sineace).

Este organismo se diseñó para establecer criterios, estándares y procesos de evaluación acreditación y certificación con el objetivo de garantizar niveles básicos de calidad en las instituciones educativas. El Sineace fomentó procesos orientados a crear una cultura de evaluación permanente de las universidades, pero esos procesos eran, en su mayoría, voluntarios. Así, la creación del Sineace representó un primer intento para establecer un sistema de aseguramiento de la calidad de la educación superior a cargo del gobierno central.

\section{El marco institucional vigente}

Debido a la creciente preocupación por la calidad del servicio educativo en las universidades del Perú, sobretodo de las universidades privadas relativamente nuevas y con precios por dejabo del promedio, se generó el escenario para llevar a cabo la reforma que es la que se encuentra vigente en el país. Esta se inició el año 2012 con la prohibición de la creación y el otorgamiento de autorizaciones para el funcionamiento de nuevas universidades y filiales por un período de 5 años, mientras se replanteaba la política de educación universitaria y se elaboraba una nueva legislación. Así, en el año 2014 se promulgó la Nueva Ley Universitaria.

El objetivo de la reforma planteada en esta ley es ordenar el mercado de educación superior universitaria. Para ello, la ley contiene un marco normativo para la creación, funcionamiento, supervisión y cierre de universidades, y del mismo modo, para la promoción de la mejora continua de la calidad de los servicios educativos universitarios. La ley señala explícitamente al Ministerio de Educación (MINEDU) como el órgano rector del aseguramiento de la calidad de la educación superior $y$, a diferencia del marco institucional anterior, se reconoce que el Estado es quien debe garantizar que el servicio público de la educación universitaria se preste en la cantidad necesaria y con un nivel de calidad aceptable. En este sentido, una primera medida fue el cierre de la Asamblea Nacional de Rectores (ANR) y del Conafu, entidades involucradas en la educación superior universitaria.

Adicionalmente, se creó la Superintendencia Nacional de Educación Superior Universitaria (Sunedu), como un organismo público técnico especializado adscrito al MINEDU. La Sunedu cuenta con autonomía técnica, funcional económica, presupuestal y administrativa, para el ejercicio de sus funciones. La Sunedu tiene como finalidad supervisar la calidad del servicio brindado por las universidades. Una de sus principales funciones es la de verifcar el cumplimiento de condiciones básicas de calidad por parte de las universidades para poder autorizar su funcionamento.

Estas condiciones básicas de calidad (CBCs) están referidas a: i) la existencia de objetivos académicos, grados, títulos y planes de estudio, ii) oferta educativa compatible con los instrumentos de planeamiento, iii) infraestructura y equipamientos adecuados para cumplir sus funciones, iv) líneas de investigación, v) disponibilidad de personal docente calificado con nomenos del $25 \%$ de docentes a tiempo completo, vi) servicios educacionales complementarios básicos, vii) mecanismos de inserción laboral, y viii) transparencia de universidades.

La verificación de las CBCs son el elemento principal del Modelo de Licenciamiento y su implementación en el sistema universitario peruano y considera los siguientes elementos clave: (i) Revisión documentaria que sustente el cumplimiento de las $\mathrm{CBCs}$, (ii) Verificación presencial del cumplimiento de las CBCs, (iii) Requerimiento de Plan de Adecuación que considere actividades, cronograma y presupuesto para alcanzar el cumplimiento de las CBCs, según corresponda.

Así, entre diciembre del 2015 y diciembre del 2017, 141 universidades y cuatro escuelas de posgrado presentaron su Solicitud de Licenciamiento Institucional (SLI) ante Sunedu. A la fecha de realización de este estudio, 72 universidades y dos escuelas de 10 posgrado han recibido su Licencia de Funcionamiento para ofrecer el servicio educativo superior universitario, luego de que la Sunedu verificara el cumplimiento de las CBCs. El proceso de licenciamiento ha estado acompañado, en algunos casos, de un sinceramiento de la oferta educativa.

Es decir, se ha otorgado el licenciamiento a algunas universidades luego de ordenar el cierre de programas específicos $y / o$ filiales. El número de universidades con licencia denegada asciende a 10 a la fecha y corresponden todas al ámbito privado.En adición a la verificación de las CBCs, Sunedu también tiene la responsabilidad de fiscalizar que los recursos públicos y los beneficios tributarios otorgados a las universidades sean destinados hacia fines educativos. Sunedu también se encarga de la administración del Registro Nacional de Grados y Titulos, y tiene la facultad de sancionar a las universidades que incumplan con las obligaciones establecidas en la ley y sus normas conexas.

Las resoluciones de la Sunedu ordenando el cierre de programas, filiales, o denegando el licenciamiento a algunas universidades, vienen generando una preocupación creciente repecto del futuro de los estudiantes cuyos programas, filiales o universidades no cumplen con la CBCs. Aún cuando Sunedu establece un período de hasta 2 años para cerrar un programa, una filial, o una universidad, y se han establecido mecanismos para procurar el traslado de los estudiantes hacia otro programas y/o universidades, la permanencia de los estudiantes universitarios puede verse afectada significativamente si no se establecen medidas efectivas para garantizar el adecuado traslado de los estudiantes, reduciendo la formación de capital humano, aspecto que es de vital importancia para incrementar la productividad y calidad de vida en una economía en desarrollo como la peruana. En este contexto se hace particularmente necesario contar con evidencia sobre los 
factores que influyen en la permanencia de los estudiantes universitarios.

\section{Un modelo sencillo de decisiones de permanencia universitaria}

La literatura teórica y empírica sobre las decisiones de inversión en capital humano y educación es amplia. Ello debido a que la educación es muy importante en muchas dimensiones. A nivel individual, la educación afecta las perspectivas de ingreso, la empleabilidad y la posibilidad de superar las limitaciones de crecer en un entorno vulnerable. También afecta la salud, la estructura familiar futura, asi como otros aspectos de la vida. A nivel nacional, el stock de capital humano y habilidades es crucial para la productividad y el crecimiento económico. La distribución de habilidades también es un factor importante para explicar la desigualdad, y la relación de las habilidades de una persona respecto de sus antecedentes familiares es clave para entender el grado de mobilidad social o intergeneracional (Burgess, 2016).

En esta sección se presenta un modelo de decisiones de permanencia en la educación universitaria y de trabajo siguendo a de Janvry et al. (2005). Se asume que el estudiante universitario maximiza el valor presente del flujo de utilidades a lo largo de su vida, eligiendo en cada periodo si continuar en la universidad (permanencia) o dejar la universidad e ingresar al mercado laboral de manera exclusiva. El proceso de decisión dura un número finito de años hasta que el estudiante alcanza el máximo nivel de educación (a nivel de pregrado, por ejemplo). Luego de ello, ingresar al mercado laboral es la única opción.

Consideremos un estudiante universitario que ha culminado el año $g$, menor al número máximo de años ofrecidos en la universidad. El estudiante puede elegir retirarse de la universidad e ingresar al mercado laboral con la formación alcanzada de $g$ años, o matricularse para el año $g+1$. Si completa satisfactoriamente el año $g+1$, enfrentará la siguiente ronda de decisiones con la formación completa hasta $g+1$. Si no aprueba el año, puede retirarse de la universidad o repetir el año. Es posible incorporar un límite para los años desaprobados, por simplicidad se asume que luego de un segundo año desaprobado, el estudiante universitario no intentará repetir el año.

La principal recompensa de educarse es la generación de oportunidades de empleo. Así, se asume que el tipo de empleo y el posible salario son funciones del grado de educación universitario alcanzado. Sean $\pi_{j}\left(x^{e}, g\right)$ y $w_{j}^{*}\left(x^{w}, g\right)$ la probabilidad de obtener un empleo del tipo $j$ y el valor presente descontado condicional de los ingresos en el empleo $j$, respectivamente, expresadas como funciones de características individuales, $x^{e}$, y características regionales $x^{w}$, y el grado de educación universitaria alcanzado $g$. El valor esperado del flujo de ingresos al abandonar la universidad con el grado completo $g$ es:

$$
W_{g}^{*}(x)=\sum_{j} \pi_{j}\left(x^{e}, g\right) w_{j}^{*}\left(x^{w}, g\right)
$$

La utilidad neta de estar matriculado en el grado $g, U_{g}\left(x^{u}\right)$, incluye la utilidad directa de asistir a la universidad y la transferencia recibida, neta de los costos educativos $c_{g}\left(x^{u}\right)$, y es potencialmente función de características individuales y del hogar, y características de la universidad o de la región en la que se ubica la misma, $x^{u}$ :

$$
U_{g}\left(x^{u}\right)=u_{g}\left(x^{u}\right)+T_{g}\left(x^{u}\right)-c_{g}\left(x^{u}\right)
$$

Finalmente, se asume que la probabilidad de aprobar el grado o año $g, P_{g}\left(x^{p}\right)$, depende de las características individuales del estudiante universitario y de su hogar. Dos decisiones determinan la dinámica de la permanencia en la universidad: (i) la decisión de repetir en casos de no aprobar, y (ii) la decisión de permanecer luego de aprobar. Para analizar la primera decisión, denotemos por $E V_{g+1}(x)$ el valor esperado del flujo de utilidades de un estudiante con grado completo $g+1$, donde $\mathrm{x}$ incluye los elementos de $x^{u}, x^{p}, x^{e}$ y $x^{w}$. Consideremos también el caso general donde la utilidad esperada directa de repetir en caso de no aprobar, $U_{g+1}^{R}$, es distinta de la utilidad esperada de haberse inscrito la primera vez $U_{g+1}$. La decisión de repetir un año desaprobado compara el retorno esperado de repetir:

$$
U_{g+1}^{R}+P_{g+1} E V_{g+1}+\left(1-P_{g+1}\right) W_{g}^{*}
$$

con el ingreso esperado $W_{g}^{*}$ que puede ser obtenido por ingresar al mercado laboral con grado $g$. Es decir, la decisión de repetir el año es tomada luego de que el estudiante identifica sus fallas y depende de la mejor predicción posible de los beneficios futuros. Asl, la decisión de repetir puede expresarse como:

$$
\begin{aligned}
& R_{g+1}=\operatorname{Pr}(\text { repetir } g+1)= \\
& \operatorname{Pr}\left(U_{g+1}^{R}+P_{g+1} E V_{g+1}+\left(1-P_{g+1}\right) W_{g}^{*}-W_{g}^{*} \geq \varepsilon_{g}\right)
\end{aligned}
$$

donde $\varepsilon_{g}$ representa el componente no observable de la utilidad o ingresos esperados que forman parte de la decisión del estudiante universitario.

De manera similar, la decisión de continuar en la universidad en el año $g+1$ luego de aprobar el año $g$ compara las utilidades esperadas bajo la decisión de permanecer con la decisión de no hacerlo. Denotemos con $E\left(V_{g} \mid s_{g+1}\right)$ a la utilidad esperada condicional en la decisión de permanencia $\left(s_{g+1} \in\{0,1\}\right)$. La utilidad esperada de permanecer en el grado $g+1$ es:

$$
\begin{aligned}
& E\left(V_{g} \mid s_{g+1}=1\right)= \\
& U_{g+1}+P_{g+1} E V_{g+1}+\left(1-P_{g+1}\right) \times \\
& \left\{R_{g+1} E\left[U_{g+1}^{R}+P_{g+1} E V_{g+1}+\left(1-P_{g+1}\right) W_{g}^{*} \mid \text { repite }\right]+\left(1-R_{g+1}\right) W_{g}^{*}\right\}
\end{aligned}
$$

La utilidad esperada futura incluye tres términos. El primer término, $P_{g+1} E V_{g+1}$, es la probabilidad de aprobar multiplicada por la utilidad con un grado adicional aprobado. El segundo término, $\left(1-P_{g+1}\right)\left\{R_{g+1} E\left[U_{g+1}^{R}+P_{g+1} E V_{g+1}+\left(1-P_{g+1}\right) W_{g}^{*} \mid\right.\right.$ repite $\left.]\right\}$, es el retorno esperado de no aprobar y repetir. El tercer término, $\left(1-P_{g+1}\right)\left(1-R_{g+1}\right) W_{g}^{*}$, es el retorno esperado de no aprobar y no repetir. La utilidad esperada de no permanecer en la universidad es:

$$
E\left(V_{g} \mid s_{g+1}=0\right)=W_{g}^{*}
$$


De manera similar a la decisión de repetir el año/grado, las decisiones en este caso son basadas en las predicciones del estudiantes universitario sobre sus beneficios futuros. Así, la decisión de permanecer en la universidad puede escribirse como:

$$
S_{g+1}=\operatorname{Pr}\left(s_{g+1}=1\right)=\operatorname{Pr}\left[U_{g+1}+B F_{g+1}-W_{g}^{*} \geq \mu_{g}\right]
$$

donde $\mu_{g}$ representa los elementos no observables en esta decisión.

De acuerdo con el modelo propuesto, las ecuaciones de comportamiento a ser estimadas son las correspondientes a la decisión de repetir luego de desaprobar, y la elección de continuar en la universidad luego de aprobar. Sin embargo, para efectos de esta investigación, el interés recae sobre la permanencia en general, de manera que, tal como se verá en la secciones siguientes, el modelo considerará la permanencia como la unión de los dos eventos descritos en el modelo.

Es decir, un estudiante universitario permanece en la universidad ya sea repitiendo el grado en caso de no haber aprobado, o cursando el grado inmediato superior en caso de haber aprobado. Adicionalmente, una característica usual respecto de la dinámica de la educación universitaria, es que los estudiantes compartan sus labores de estudio con algún empleo. Este elemento, también será tomado en cuenta en la estrategia empírica que se describe en la siguiente sección.

\section{Estrategia empírica}

En esta sección se describe la estrategia para abordar el objetivo del presente estudio, la base de datos utilizada, la estadística descriptiva y los modelos de elección discreta de datos en panel.

\section{Base de datos}

La base de datos utilizada es un panel de dos periodos y se elaboró en base a 3 diferentes fuentes: (1) la Encuesta Nacional de Hogares Panel Actualizada 20132017, para la construcción de las variables asociadas a las características del hogar y del estudiante, (2) la información disponible del Observatorio de Educación y Empleo "Ponte en Carrera", para la construcción de las variables como el costo anual por carreras y universidades y (3) la información administrada por la Sunedu sobre los resultados del proceso de licenciamiento, para obtener la lista de universidades que garantizaron las condiciones básicas de calidad para la obtención de su autorización de funcionamiento institucional.

\section{Estadística descriptiva}

De acuerdo con lo señalado en secciones previas, los factores asociados al acceso y permanencia en la educación superior están relacionados con características socioeconómicas del estudiante y del hogar. Adicional a ello, para efectos de esta investigación se consideran características de la oferta educativa. Las variables del modelo se listan en la Tabla ??.

TABLA 1
En la Tabla ?? se muestran los principales estadísticos descriptivos de las variables del modelo, los cuales servirán para identificar potenciales diferencias y relaciones antes de observar los resultados de las regresiones. El número de estudiantes de la muestra asciende a 796 y el número de observaciones a 1,592 dado que se tiene un panel de 2 periodos. Como se aprecia en la Tabla 2, en el periodo 20162017 se presenta altos niveles de permanencia en educación universitaria.

La mayor parte de la muestra son estudiantes que viven en Lima Metropolitana, solteros y que pertenecen a un hogar donde el jefe de hogar por lo menos culminó los estudios de secundaria. Asimismo, se puede notar que el porcentaje de alumnos estudiando en universidades que acreditaron las condiciones básicas de calidad ascendió a $57.3 \%$. Por otro lado, explorando algunos factores asociados con los niveles de permanencia que mostraron variación positiva, tenemos la condición de aprobado del estudiante y el ingreso del hogar. Otras variables como la lengua materna, la edad, carga familiar y condición de pobreza fueron exploradas inicialmente; sin embargo, por presentar poca variabilidad en los datos de la muestra no fueron consideradas.

\section{TABLA 2}

En la Tabla ?? se observan las características de los individuos, según el tipo de gestión de la universidad (pública o privada) para el año 2017. Se observa que la masa estudiantil que no deserta es mayor en las universidades públicas que en las privadas. Por otro lado, algunas características de los estudiantes de universidades privadas son resaltantes: los jefes de su hogar tienen mayor número de años de educación, presentan mayores niveles de ingreso del hogar y trabajan más horas a la semana.

El hogar que presenta mayor nivel de ingreso puede estar en la capacidad de dar soporte económico al estudiante, lo cual podría relacionarse con la decision de seguir estudiando. Asimismo, otro factor que sustentaría esta decisión está relacionado al trabajo de un grupo importante de estudiantes, solventando ellos mismos sus estudios.

TABLA 3

En Tabla ?? se detallan las características de los estudiantes, según condición de la universidad a la que asiste (con o sin licencia institucional) en el año 2017. De esta manera, se muestra que la proporción de alumnos de universidades con licencia institucional es mayor que la proporción de alumnos en universidades no licenciadas. También se puede observar que el costo mensual de las universidades no licenciadas es menor, aspecto que podría estar relacionado con la calidad que ofrecen estos centros de estudios.

\section{TABLA 4}

A partir de esta primera exploración de los datos, se observa que los niveles de permanencia en la educación universitaria son altos. Sin embargo, en el marco del proceso de licenciamiento institucional de las universidades iniciado en el 2015, bajo el cual existe la certeza del cierre de universidades, programas y filiales que no cumplan con acreditar las condiciones básicas de calidad, dichos niveles 
podrian variar en la medida que las decisiones de los estudiantes se vean afectadas en este contexto.

Así, se plantea identificar los factores o determinantes de la permanencia en la educación superior universitaria a través de una aproximación econométrica, teniendo en cuenta algunas variables de la oferta educativa como el tipo de gestión de la universidad, el costo mensual del servicio y el otorgamiento de la licencia institucional que garantiza condiciones básicas de calidad, además de características de los estudiantes y sus hogares.

\section{Aproximación econométrica}

En esta subsección se describe el modelo económetrico para examinar e identificar cuáles son los factores relevantes de la permanencia de los estudiantes universitarios. De acuerdo con el modelo teórico propuesto, existen 3 tipos de factores potenciales: factores a nivel de individuo, factores a nivel de hogar y factores a nivel de universidad. Adicional a los dos primeros grupos, factores que han sido utilizados en otros estudios, se ha considerado agregar factores a nivel de universidad para analizar las posibles relaciones o efectos parciales sobre la permanencia universitaria.

Para ello se plantea el uso de modelos de datos en panel de efectos fijos y efectos aleatorios: los modelos probabilísticos probit y logit. Estos modelos de probabilidad no lineal presentan variable dependiente discreta y permiten medir los efectos parciales de las variables independientes sobre la probabilidad de permanecer en la educación universitaria. La forma funcional de estos modelos es:

$$
P(y=1 \mid x, z, w)=G(x \beta+z \gamma+w \delta)
$$

donde $y$ es la variable dependiente binaria que toma el valor de 1 si el estudiante permanece en la universidad y 0 en otro caso, $x, z, w$ representan el conjunto de factores potenciales descritos líneas arriba, $G(:)$ es la función de distribución acumulada (logística o normal) y toma el valor entre 0 y 1 . De acuerdo con los tipos de factores incluidos en nuestro modelo, se plantean las siguientes especificaciones:

$$
\begin{gathered}
P(y=1 \mid x)=G(x \beta) \\
P(y=1 \mid x, z)=G(x \beta+z \gamma) \\
P(y=1 \mid x, z, w)=G(x \beta+z \gamma+w \delta)
\end{gathered}
$$

En la ecuación (7) la especificación presentada incluye como variables explicativas sólo a aquellas relacionadas con las características individuales del estudiante, $x$ : la condición civil, si el estudiante fue aprobado el periodo anterior, las horas de trabajo y la condición de bancarización del individuo. La ecuación (8) adiciona las variables características del hogar del estudiante, $z$ : el ingreso mensual del hogar, la ubicación geográfica y el número de años del jefe de hogar.

Asimismo, en la ecuación (9) se agregan al modelo los factores que podrían estar relacionados con la calidad del servicio que ofrece la universidad, $w$ : el tipo de gestión de la universidad, la condición de licencia institucional y el costo mensual del servicio educativo. Por último, se plantea una cuarta aproximación, que consiste en agregar a la ecuación (9) una interacción de las variables licencia y costo mensual.

\section{Resultados del análisis econométrico}

El punto de partida del análisis econométrico fue realizar el contraste del Test de Hausman a la primera especificación, a fin de evaluar el modelo más apropiado a la muestra del estudio. Dado que no se rechaza la hipótesis nula del test, es conveniente utilizar el modelo de efectos aleatorios. Si bien el modelo de efectos aleatorios implica supuestos fuertes, en el presente estudio resulta útil dado que se planteó explorar la importancia de factores de la permanencia universitaria,incluyendo a aquellos que no varían en el tiempo como el género, y aquellos con muy poca variabilidad para la muestra de, como la condición civil y la ubicación geográfica del estudiante.

En la Tabla ?? se presentan los resultados de las regresiones realizadas entorno a las especificaciones descritas en la sección anterior. Se obtienen 4 regresiones para los modelos con especificación logit. Los resultados del primer modelo (1) sugieren que la probabilidad de permanecer en la universidad está fuertemente relacionada de manera positiva con la condición de aprobado del estudiante, lo que se relaciona con el modelo teórico descrito y la evidencia que sugiere la estadistica descriptiva. Asimismo, la variable horas de trabajo muestra la presencia de efectos negativos en la permanencia de estudiantes universitarios.

\section{TABLA 5}

Estos factores mantienen su nivel de importancia en los resultados de la segunda especificación. En los dos últimos modelos, donde se toman en cuenta las variables que expresan las características de la universidad, los resultados sugieren que el costo mensual del servicio educativo y que la universidad sea de gestión pública aumenta la probabilidad de la permanencia universitaria. Respecto de la condición del cumplimiento de las condiciones básicas de calidad por parte de la universidad, éste no parece ser un factor significativo; se registra un cambio de signo del coeficiente de negativo a positivo.

Si bien los signos de los coeficientes pueden ser un indicativo del tipo de relación de los factores con la variable de permanencia universitaria, en los modelos de probabilidad no lineal se requiere realizar el cálculo de los efectos marginales.

En la Tabla ?? se muestra que la condición de aprobado es un factor significativo e incrementa la probabilidad de permanencia en tres puntos porcentuales; esto indica que los estudiantes que aprueban el periodo académico anterior son más propensos a permanecer en las universidades. Se evidencia también que las horas que dedica el estudiante a trabajar (trabajo remunerado y no remunerado) es un factor significativo que presenta una relación negativa con la variable dependiente, aunque la magnitud del efecto marginal es reducida.

Esto podría deberse a que la decisión de asignar horas de trabajo no es del todo excluyente para el estudiante; la realidad de la universidad peruana está caracterizada por contar con muchos estudiantes que asisten a las aulas y a la 
vez trabajan, en particular es el caso de los estudiantes de universidades privadas, quienes poseen un mayor número promedio de horas de trabajo a la semana en comparación con los de universidades públicas.

\section{TABLA 6}

Respecto a las variables relacionadas con la oferta educativa, el tipo de gestión de la universidad y el costo mensual son factores que inciden significativamente en la permanencia universitaria, los efectos marginales de ambas variables tienen signo positivo y son significativos. En el primer caso, pertenecer a una universidad pública incrementa la permanencia en la universidad en alrededor de cuatro puntos porcentuales.

Respecto del costo mensual, se debe entender que de acuerdo con el contexto institucional descrito que motivó la reforma universitaria (proliferación de universidades privadas nuevas de bajo costo y calidad) y la dimensión de variabilidad de los datos (variación transversal), esta variable es un proxy de la calidad del servicio educativo. En ese sentido, aún cuando el efecto marginal sea reducido, se encuentra evidencia de que una mayor calidad del servicio educativo contribuye a incrementar la permanencia universitaria.

Finalmente, nótese que la obtención de la licencia institucional no estaría incidiendo en la permanencia universitaria. Esto se explica a que, como se describió previamente, la obtención de la licencia está condicionada a la verificación de condiciones básicas de calidad y la calidad de la oferta educativa se ve mejor reflejada en el costo de las universidades. Por lo demás, el proceso de licenciamiento es un proceso que se encuentra en curso y será de mucho interés evaluar posteriormente sus efectos en un conjunto de variables relacionadas con el desempeño del sistema universitario peruano, incluyendo los efectos sobre la permanencia de los estudiantes.

\section{Conclusiones}

En esta investigación se presenta un análisis empírico sobre las decisiones de permanencia de los estudiantes universitarios en el marco de la reforma universitaria que se viene implementando en el Perú desde el año 2014. Un aspecto central de esta reforma es la creación de la Sunedu y su labor en relación al otorgamiento de licencias institucionales previa verificación de hasta ocho condiciones básicas de calidad (CBCs).

Así, a través de distintas especificaciones para modelos de elección discreta de datos de panel con información de los años 2016 y 2017, se encuentra evidencia interesante sobre los factores que estarian afectando las decisiones de permanencia. Especificamente, se muestra evidencia de que tanto el desempeño de los estudiantes universitarios como la intensidad de su participación en el mercado laboral tienen efectos significativos en la probabilidad de permanencia.

Adicionalmente, características de la oferta educativa relacionadas con la gestión de la universidad y el costo académico también tienen efectos que son estadísticamente significativos en la probabilidad de permanencia de los estudiantes universitarios. En el contexto del análisis propuesto, las diferencias en los costos académicos son una buena medida de las diferencias en las condiciones de la calidad del servicio educativo y en ese sentido, los resultados sugieren evidencia de que una mayor calidad del servicio educativo contribuye positivamente a la permanencia de los estudiantes universitarios, aspecto que está directamente relacionado con los objetivos de la reforma universitaria y la verificación de condiciones básicas de calidad (CBCs) para el otorgamiento de licencias institucionales de funcionamiento de las universidades en el Perú.

Esta investigación es la primera que analiza las decisiones de permanencia en el contexto de la reforma universitaria en Perú $y$, a pesar de las limitaciones que se tienen (disponibilidad de información y potenciales sesgos por endogeneidad), abre un conjunto interesante de posibilidades de investigaciones futuras para contribuir con un análisis consistente a la discusión sobre estos temas relevantes y, eventualmente, a la implementación de políticas efectivas relacionadas con la permanencia de los estudiantes universitarios.

En línea con lo señalado, el proceso de reforma universitaria va a ir generando información relacionada con indicadores de calidad especificos, de manera que será posible monitorear las distintas dimensiones de la calidad de los servicios educativos y realizar análisis del impacto de las políticas públicas sobre el desempeño del sistema universitario peruano, incluyendo los impactos sobre la permanencia.

La utilización de registros administrativos sobre los estudiantes universitarios con el objetivo de contar con un marco muestral para el levantamiento de información con mayor precisión y representatividad también es un aspecto que puede contribuir a un mejor entendimiento de las decisiones de permanencia, así como el desempeño de los estudiantes y egresados universitarios.

\section{Agradecimientos}

La autora agradece al apoyo de la Escuela Profesional de Economía y del Instituto de Investigación de la Facultad de Ciencias Contables y Económicas y Financiceras de la Universidad de San Martín de Porres.

\section{Referencias}

Becker, Gary S. (1994). Human Capital: A Theoretical and Empirical Analysis, with Special Reference to Education. Third Edition. NBER.

Benavides, M., León, J., Haag, F., y Cueva, S. (2015). Expansión y diversificación de la educación superior universitaria y su relación con la desigualdad y la segregación. Lima: Grupo de Análisis para el Desarrollo.

British Council (2016). La reforma del Sistema Universitario Peruano:Internacionalización, avance, retos y oportunidades.

Burgess, Simon (2016). Human Capital and Education: The State of the Art of the Economics of Education. IZA DP No 9885.

Del Pozo Loayza, C. E. (2018). Efectos de la desregulación del sistema universitario en el mercado laboral en Perú (Doctoral dissertation, Facultad de Ciencias Económicas de la Universidad Nacional de La Plata). 
de Janvry, A.; Finan, F.; y E. Sadoulet (2005). Using a structural model of educational choice to improve efficiency. Working Paper, Department of Agricultural and Resource Economics, University of California at Berkeley.

Ferreyra, María Marta, Ciro Avitabile, Javier Botero Álvarez, Francisco Haimovich Paz, and Sergio Urzúa (2017). At a Crossroads: Higher Education in Latin America and the Caribbean. Directions in Development. Washington, DC: World Bank.

Guerrero, G. and V. Rojas (2019). Young women and higher education in Peru: how does gender shape their educational trajectories?, Gender and Education, DOI: 10.1080/09540253.2018.1562055.

Pasco-Font, A.; y J. Saavedra (2001). Reformas estructurales y bienestar: Una mirada al Perú de los noventa. Lima: Grupo de Análisis para el Desarrollo.

Rodríguez, J., and Montoro, L. (2013). La educación superior en el Perú: Situación actual y perspectivas. (No. 2013370). Departamento de Economía-Ponticia Universidad Católica del Perú.

Sánchez, A., and Singh, A. (2018). Accessing higher education in developing countries: Panel data analysis from India, Peru, and Vietnam. World Development, 109, 261-278.

Schofer, E., and Meyer, J. W. (2005). The worldwide expansion of higher education in the twentieth century. American Sociological Review, 70(6),898-920.

Superintendencia Nacional de Educación Superior Universitaria. (2015). El modelo de licenciamiento y su implementación en el Sistema Universitario Peruano. Lima: Sunedu.

Sunedu (2018). Informe bienal sobre la realidad universitaria peruana. 1ra Edición.

Cuadro 1. Variables incluidas en el modelo

\begin{tabular}{|c|c|}
\hline Variable & Descripcion \\
\hline aprobado & $\begin{array}{l}1 \text { si el estudiante aprobo el ciclo academico } \\
\text { anterior y } 0 \text { en otro caso }\end{array}$ \\
\hline soltero & 1 si el estudiante es soltero y 0 en otro caso \\
\hline mujer & 1 si el estudiante es mujer y 0 en otro caso \\
\hline lima & $\begin{array}{l}1 \text { si el estudiante vive en Lima Metropolitana y } 0 \\
\text { en otro caso }\end{array}$ \\
\hline hrstrab & $\begin{array}{l}\text { Numero de horas de trabajo a la semana del } \\
\text { estudiante (actividad principal y secundarias) }\end{array}$ \\
\hline banc & 1 si el estudiante esta bancarizado y 0 en otro caso \\
\hline lingresom & logaritmo del ingreso mensual del hogar \\
\hline naejef & numero de años de educacion del jefe de hogar \\
\hline licencia & $\begin{array}{l}1 \text { si el estudiante asiste a una universidad con } \\
\text { licencia institucional y } 0 \text { en otro caso }\end{array}$ \\
\hline tipou & $\begin{array}{l}1 \text { si el estudiante asiste a una universidad publica y } \\
0 \text { si el estudiante asiste a una universidad privada }\end{array}$ \\
\hline costomensual & costo mensual en soles por universidad \\
\hline perma & $\begin{array}{l}1 \text { si el estudiante permanece en la educacion } \\
\text { universitaria y } 0 \text { en otro caso }\end{array}$ \\
\hline
\end{tabular}

Cuadro 2. Estadıstica descriptiva: Muestra total

\begin{tabular}{lcccc}
\hline \hline & \multicolumn{2}{c}{ Total 2016 } & \multicolumn{2}{c}{ Total 2017 } \\
& media & sd & media & sd \\
\hline perma & 0.92 & 0.27 & 0.92 & 0.27 \\
soltero & 0.91 & 0.29 & 0.90 & 0.30 \\
aprobado & 0.93 & 0.26 & 0.94 & 0.23 \\
mujer & 0.51 & 0.50 & 0.51 & 0.50 \\
lima & 0.92 & 0.27 & 0.92 & 0.27 \\
naejef & 14.27 & 4.40 & 14.28 & 4.46 \\
ingresom & 4581.70 & 3651.88 & 4635.27 & 3755.60 \\
hrstrab & 15.34 & 20.47 & 14.82 & 20.08 \\
banc & 0.62 & 0.49 & 0.59 & 0.49 \\
Licencia & 0.06 & 0.23 & 0.57 & 0.49 \\
costomensual & 296.88 & 332.60 & 296.88 & 332.60 \\
tipou & 0.41 & 0.490 .41 & 0.49 & \\
\hline$N$ & 796 & \multicolumn{4}{c}{796} \\
\hline \hline
\end{tabular}

Elaboracion propia

Cuadro 3. Estadıstica descriptiva: Por tipo de universidad-2017

\begin{tabular}{lcccc}
\hline \hline & \multicolumn{2}{c}{ U. Publicas } & \multicolumn{2}{c}{ U. Privadas } \\
& mean & sd & mean & sd \\
\hline perma & 0.94 & 0.22 & 0.90 & 0.29 \\
soltero & 0.91 & 0.27 & 0.88 & 0.32 \\
aprobado & 0.93 & 0.25 & 0.94 & 0.21 \\
mujer & 0.45 & 0.49 & 0.54 & 0.49 \\
lima & 0.95 & 0.21 & .90 & 0.29 \\
naejef & 13.53 & 4.46 & 14.79 & 4.38 \\
ingresom & 3624.52 & 2470.03 & 5329.08 & 4293.93 \\
hrstrab & 11.88 & 17.52 & 16.83 & 21.43 \\
banc & 0.67 & 0.46 & 0.52 & 0.49 \\
Licencia & 0.61 & 0.48 & 0.54 & 0.49 \\
costomensual & 8.37 & 3.55 & 494.91 & 300.25 \\
\hline$N$ & 324 & \multicolumn{4}{c}{472} \\
\hline \hline
\end{tabular}

Elaboracion: propia

Cuadro 4. Estadıstica descriptiva: Por licenciamiento- 2017

\begin{tabular}{lcccc}
\hline \hline & \multicolumn{3}{c}{ U. Licenciadas } & \multicolumn{2}{c}{ U. No Licenciadas } \\
& mean & sd & mean & sd \\
\hline perma & 0.95 & 0.22 & 0.89 & 0.31 \\
soltero & 0.93 & 0.26 & 0.86 & 0.35 \\
aprobado & 0.94 & 0.24 & 0.94 & 0.23 \\
mujer & 0.48 & 0.50 & 0.55 & 0.50 \\
lima & 0.92 & 0.27 & 0.92 & 0.27 \\
naejef & 14.66 & 4.29 & 13.78 & 4.65 \\
ingresom & 5070.13 & 4345.19 & 4052.05 & 2672.11 \\
hrstrab & 12.774 & 18.68 & 17.57 & 21.53 \\
banc & 0.56 & 0.50 & 0.63 & 0.48 \\
tipou & 0.43 & 0.50 & 0.37 & 0.48 \\
costomensual & 355.71 & 391.18 & 217.97 & 207.72 \\
\hline$N$ & 456 & \multicolumn{4}{c}{340} \\
\hline \hline Elaboracion propia &
\end{tabular}


Cuadro 5. Estimaciones de la probabilidad de permanencia

\begin{tabular}{|c|c|c|c|c|c|c|c|c|c|}
\hline & $\begin{array}{c}(1) \\
\text { Logit1 }\end{array}$ & $\begin{array}{c}(2) \\
\text { Logit2 } \\
\end{array}$ & $\begin{array}{c}(3) \\
\text { Logit3 } \\
\end{array}$ & $\begin{array}{c}(4) \\
\text { Logit4 }\end{array}$ & & & & & \\
\hline \multirow[t]{2}{*}{$\begin{array}{l}\text { perma } \\
\text { soltero }\end{array}$} & 0.418 & 0.462 & 0.366 & 0.371 & \multicolumn{5}{|c|}{ Cuadro 6. Efectos marginales en la probabilidad de permanencia } \\
\hline & $(0.94)$ & $(1.02)$ & $(0.84)$ & (0.85) & & (1) & (2) & (3) & (4) \\
\hline aprobado & $1.176^{* *}$ & $1.167^{* *}$ & $1.104^{* *}$ & $1.105^{* *}$ & & Logit1 & Logit2 & Logit3 & Logit4 \\
\hline & $(2.83)$ & $(2.75)$ & $(2.63)$ & $(2.62)$ & soltero & $\begin{array}{c}0.0118 \\
(0.94)\end{array}$ & $\begin{array}{l}0.0131 \\
(1.03)\end{array}$ & $\begin{array}{c}0.0109 \\
(0.85)\end{array}$ & $\begin{array}{c}0.0110 \\
(0.85)\end{array}$ \\
\hline hrstrab & $\begin{array}{c}-0.0424^{* * *} \\
(-6.29)\end{array}$ & $\begin{array}{c}-0.0428^{* * *} \\
(-6.27)\end{array}$ & $\begin{array}{c}-0.0410^{* * *} \\
(-5.90)\end{array}$ & $\begin{array}{c}-0.0410^{* * *} \\
(-5.90)\end{array}$ & aprobado & $\begin{array}{c}0.0334^{*} \\
(2.49)\end{array}$ & $\begin{array}{c}0.0331^{*} \\
(2.45)\end{array}$ & $\begin{array}{c}0.0330^{*} \\
(2.40)\end{array}$ & $\begin{array}{c}0.0328^{*} \\
(2.40)\end{array}$ \\
\hline banc & $\begin{array}{c}0.0726 \\
(0.26)\end{array}$ & $\begin{array}{l}0.128 \\
(0.45)\end{array}$ & $\begin{array}{l}0.130 \\
(0.45)\end{array}$ & $\begin{array}{l}0.124 \\
(0.43)\end{array}$ & hrstrab & $\begin{array}{c}-0.00120^{* * *} \\
(-3.94)\end{array}$ & $\begin{array}{c}-0.00122^{* * *} \\
(-3.96)\end{array}$ & $\begin{array}{c}-0.00122^{* * *} \\
(-4.07)\end{array}$ & $\begin{array}{c}-0.00122^{* * *} \\
(-4.06)\end{array}$ \\
\hline mujer & $\begin{array}{c}-0.0683 \\
(-0.24)\end{array}$ & $\begin{array}{l}-0.102 \\
(-0.36)\end{array}$ & $\begin{array}{c}-0.0457 \\
(-0.16)\end{array}$ & $\begin{array}{c}-0.0447 \\
(-0.15)\end{array}$ & banc & $\begin{array}{c}0.00206 \\
(0.26)\end{array}$ & $\begin{array}{c}0.00364 \\
(0.44)\end{array}$ & $\begin{array}{c}0.00388 \\
(0.45)\end{array}$ & $\begin{array}{c}0.00370 \\
(0.43)\end{array}$ \\
\hline lima & & $\begin{array}{l}0.540 \\
(1.18)\end{array}$ & $\begin{array}{l}0.290 \\
(0.63)\end{array}$ & $\begin{array}{l}0.304 \\
(0.66)\end{array}$ & mujer & $\begin{array}{c}-0.00194 \\
(-0.24)\end{array}$ & $\begin{array}{c}-0.00291 \\
(-0.36)\end{array}$ & $\begin{array}{c}-0.00136 \\
(-0.16)\end{array}$ & $\begin{array}{c}-0.00133 \\
(-0.15)\end{array}$ \\
\hline naejef & & $\begin{array}{c}0.0178 \\
(0.50)\end{array}$ & $\begin{array}{c}0.0185 \\
(0.53)\end{array}$ & $\begin{array}{c}0.0190 \\
(0.54)\end{array}$ & lima & & $\begin{array}{c}0.0153 \\
(1.14)\end{array}$ & $\begin{array}{c}0.00865 \\
(0.62)\end{array}$ & $\begin{array}{c}0.00902 \\
(0.65)\end{array}$ \\
\hline lingresom & & $\begin{array}{l}0.208 \\
(1.01)\end{array}$ & $\begin{array}{l}0.154 \\
(0.71)\end{array}$ & $\begin{array}{l}0.156 \\
(0.72)\end{array}$ & naejef & & $\begin{array}{c}0.000504 \\
(0.50)\end{array}$ & $\begin{array}{c}0.000552 \\
(0.53)\end{array}$ & $\begin{array}{c}0.000563 \\
(0.54)\end{array}$ \\
\hline Licencia & & & $\begin{array}{l}-0.191 \\
(-0.71)\end{array}$ & $\begin{array}{c}0.0288 \\
(0.07)\end{array}$ & lingresom & & $\begin{array}{c}0.00592 \\
(0.99)\end{array}$ & $\begin{array}{c}0.00460 \\
(0.71)\end{array}$ & $\begin{array}{c}0.00462 \\
(0.71)\end{array}$ \\
\hline costomensual $(\mathrm{cm})$ & & & $\begin{array}{c}0.00206^{*} \\
(2.52)\end{array}$ & $\begin{array}{c}0.00255^{* *} \\
(2.77)\end{array}$ & Licencia & & & $\begin{array}{c}-0.00570 \\
(-0.70)\end{array}$ & $\begin{array}{c}-0.00557 \\
(-0.67)\end{array}$ \\
\hline tipou & & & $\begin{array}{c}1.445^{* *} \\
(3.13)\end{array}$ & $\begin{array}{c}1.513^{* * *} \\
(3.30)\end{array}$ & $\mathrm{cm}$ & & & $\begin{array}{c}0.0000614^{*} \\
(2.26)\end{array}$ & $\begin{array}{c}0.0000696^{*} \\
(2.44)\end{array}$ \\
\hline Licencia $\times \mathrm{cm}$ & & & & $\begin{array}{c}-0.000805 \\
(-0.87)\end{array}$ & tipou & & & $\begin{array}{c}0.0431^{* *} \\
(2.70)\end{array}$ & $\begin{array}{c}0.0449^{* *} \\
(2.80)\end{array}$ \\
\hline Cons & $\begin{array}{c}3.108^{* * *} \\
(4.94) \\
\end{array}$ & $\begin{array}{l}0.631 \\
(0.36)\end{array}$ & $\begin{array}{l}0.283 \\
(0.16)\end{array}$ & $\begin{array}{l}0.117 \\
(0.06)\end{array}$ & $\mathrm{N}$ & 1592 & 1592 & 1592 & 1592 \\
\hline $\begin{array}{l}\text { Insig2u } \\
\text { _cons }\end{array}$ & $\begin{array}{c}1.293^{* * *} \\
(4.12) \\
\end{array}$ & $\begin{array}{c}1.299^{* * *} \\
(4.13)\end{array}$ & $\begin{array}{c}1.264^{* * *} \\
(3.93) \\
\end{array}$ & $\begin{array}{c}1.273^{* * *} \\
(3.98)\end{array}$ & $\begin{array}{l}{ }^{*} p<0,05 \text {, } \\
\text { Elaboracion }\end{array}$ & $\begin{array}{l}p<0,01, * * * \\
\text { Propia }\end{array}$ & $<0,001$ & & \\
\hline$N$ & 1592 & 1592 & 1592 & 1592 & & & & & \\
\hline
\end{tabular}

